\title{
Analyzing the Effect of Channel Spacing and Chromatic Dispersion Coefficient on FWM in Optical WDM System
}

\author{
Mehtab Singh \\ ECE Department, Satyam Institute of Engineering and Technology, Amritsar, \\ India \\ mehtab91singh@gmail.com
}

\begin{abstract}
In order to minimize the system impairments produced as a result of repeater circuits, optical amplifiers are utilized in wave division multiplexed (WDM) systems these days, which are capable of amplifying complete WDM signal in optical domain directly. Although WDM systems are capable of transmitting data at high data rates still, it faces many serious problems which degrade the performance of the optical transmission system such as four-wave mixing (FWM). In this paper, the effect of channel spacing in WDM systems and chromatic dispersion coefficient value on optical transmission system's performance in terms of eye diagrams and input-output spectrums is discussed. The outcomes of the simulations show that by using unequal channel spacing FWM is reduced and it can be further reduced by increasing the value of dispersion coefficient.
\end{abstract}

Keywords: Wave Division Multiplexing, Four Wave Mixing, chromatic dispersion coefficient, channel spacing

\section{Introduction}

The very high bandwidth of optical fiber has led to the development of extremely high capacity, and long-haul communication system which is best utilized along with wave division multiplexing (WDM) [1]. But various impairments like chromatic dispersion and nonlinear effects in optical fiber provide a certain limitation on the overall performance of optical communication system. In an optical fiber, the chromatic dispersion can be minimized by employing dispersion compensation fiber (DCF) along the length of optical fiber but by reducing dispersion, nonlinear effects in an optical fiber are promoted to a great extent typically four-wave mixing (FWM) [2-3]. Four wave mixing can lead to serious performance degradation of a wave division multiplexing system. FWM can cause BER fluctuations in optical networks that can lead to degradation of optical signal to noise (OSNR) and quality of service (QoS) in a WDM network having a highly complex nonlinear effect. In four wave mixing, two or more frequencies react with each other and by reacting with each other they produce multiple new frequency components of the same order as that of original frequencies. Four wave mixing is a kind of Kerr- effect in the optical domain and occurs when two or more different wavelengths travel in same optical fiber and optical power of one wavelength is transferred to optical power of adjacent wavelength. Such transfers of power not only cause power loss for the system but also cause inter-channel crosstalk which results in overall degradation of the system performance [4]. Four wave mixing is generally avoided but still it finds many applications such as transmitting data at another wavelength which can be produced as a result of interaction amongst different wavelengths. Four wave mixing effect can also be used to convert one wavelength to another as a wavelength converter. FWM can also be used to generate optical signals having inverted spectrum as that of the original signal by utilizing optical phase conjugation process. Figure 1 shows a schematic of a two and three channel four- wave mixing effect producing spurious side modes. 


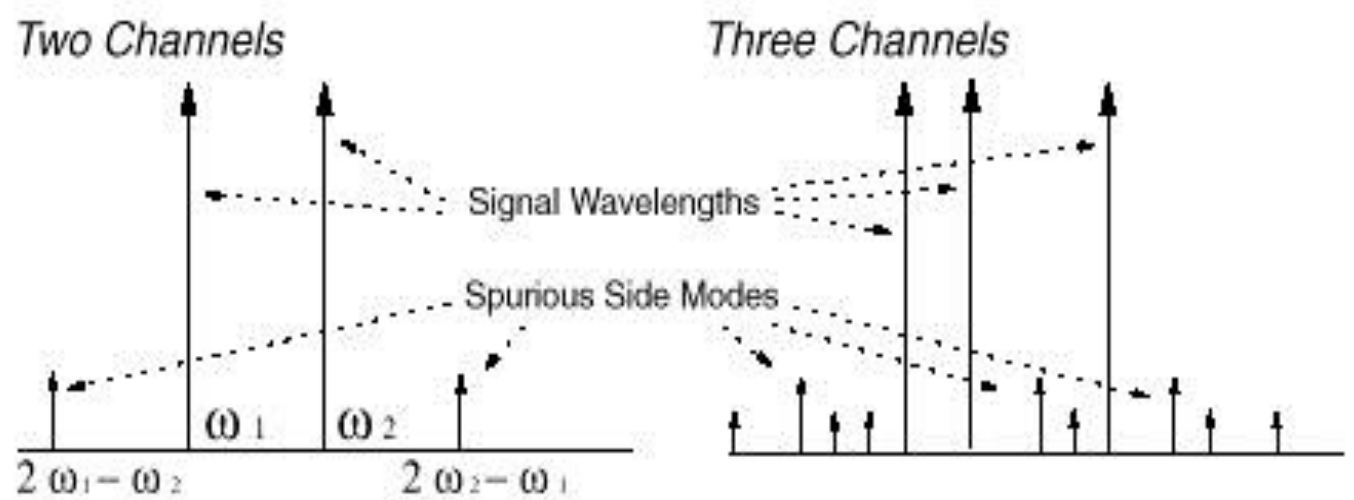

Figure 1. Four Wave Mixing in Frequency Domain [5]

If in a wave division multiplexing system there are large numbers of closely spaced wavelengths having high optical power, it can result in generation of many kinds of nonlinear effects, including:

- $\quad$ Cross-Phase Modulation (XPM)

- $\quad$ Stimulated Raman Scattering (SRS)

- $\quad$ Stimulated Brillouin Scattering (SBS)

- $\quad$ Four Wave Mixing (FWM)

These non-linear effects play a vital role in overall performance of a fiber optical communication system. Due to XPM and FWM, channels at different wavelengths interfere with each other which results in the limitation on the transmission power of each channel in a wave division multiplexing system. Transmitting information signal at high powers results not just only in promoting FWM and XPM effects but also in activating other non-linear effects such as SBS and SRS [9-10]. Four wave mixing also known as four-photon mixing poses a severe limitation on data rates and maximum repeater distance spacing in an optical fiber communication link. Due to FWM, in a multiple channel WDM system, two or more wavelengths interact with each other resulting in new frequencies also known as beat frequencies which travel at the expense of the power of original information carrying signal. The frequencies produced due to FWM fall directly in the frequency band of original information carrying signals. As a result, it is very important to minimize FWM effect in order to prevent interference between FWM frequency components and information carrying signals [11].

In this paper, the overall performance of optical WDM system is investigated taking four- wave mixing effect (FWM) into consideration. Different simulation parameters such as chromatic dispersion coefficient and WDM channel spacing are varied one at a time keeping rest of the simulation parameters constant and their effect on system performance is studied. The effect of channel spacing in a wave division multiplexing system on the efficiency of four wave mixing effect is investigated. It was seen that if channel is kept at unequal spacing the efficiency of FWM effect is reduced to a great extent in a WDM system. Also, the effect of chromatic dispersion coefficient on the efficiency of four-wave mixing effect is discussed. It was found that as the value of dispersion coefficient is increased, the efficiency of FWM effect starts falling.

The rest of the paper is organized as follows; in Section 2, theoretical background of four- wave mixing is discussed in detail. In Section 3, simulation setup and different simulation parameters used in this analysis is presented. In Section 4, results of all the simulations are discussed in detail and in Section 5, the conclusion of the analysis is presented and discussed. 


\section{Theoretical Background}

Intermodal dispersion is one of the main reasons of signal degradation in multimode fibers. Although with use of single mode fiber it is possible to eliminate intermodal dispersion, but different wavelength components present in fundamental mode of optical fiber carry with different velocities due to the fact that the speed of the information carrying signal is dependent upon the refractive index which in turn depends on wavelength of the information carrying signal [2-7]. This gives rise to a wavelength dependent group velocity delay. Due to the fact that the group velocity delay depends on the wavelength, different wavelengths will take different time to travel the same distance. As a result, the optical signal having a finite spectral width spreads in the time domain as it travels through a single mode fiber. The amount of pulse spread is very important factor in overall performance of optical transmission system and needs to be calculated. The dispersion can also be determined by taking into account waveguide dispersion and material dispersion if a precise value of dispersion is required. Thus in order to find total dispersion, material dispersion and waveguide dispersion needs to be evaluated and taken into account.

Four Wave Mixing (FWM) is a kind of non-linear effect in which three signal frequencies interact with each other in an optical fiber and produce numerous mixing products. It is the result of the dependence of refractive index of fiber on the intensity of information carrying light signal traveling along the optical fiber. If three information carrying signal with frequencies $f_{a}, f_{b}$ and $f_{c}$ are incident at the input of fiber, then new beat frequencies are generated whose frequencies is given by [8]:

$$
f_{a b c}=f_{a}+f_{b}-f_{c}
$$

where, $(\mathrm{a}, \mathrm{b}, \mathrm{c}=1,2,3)$

There is increase in number of side bands due to FWM effect and is given by [8]:

$$
\mathrm{M}=\left(\frac{N^{3}-N^{2}}{2}\right)
$$

Where $\mathrm{N}$ signifies the number of channels available and $\mathrm{M}$ denotes a total number of side bands generated. For example, ten (10) channels produce 450 side bands. Figure 2 demonstrates how FWM mixing products due to channel increases.

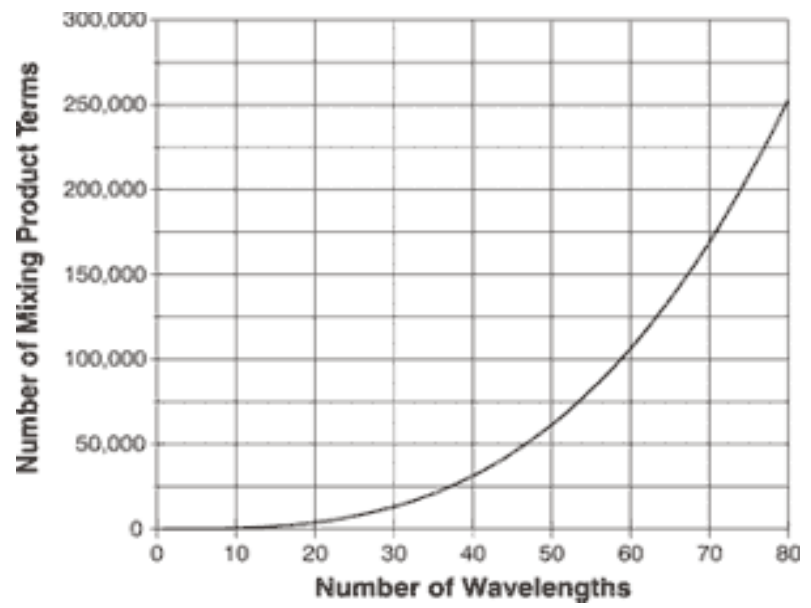

Figure 2. Number of Four-wave Mixing Products [6] 
The FWM power produced at end of optical fiber interaction of frequencies $f_{a}, f_{b}$ and $f_{c}$ is given by [2]:

$$
P_{F W M}=\frac{1024 \pi^{2}}{n^{4} \lambda^{2} c^{2}}\left[\frac{D X_{111} L_{\text {eff }}}{A_{\text {eff }}}\right]^{2} P_{a \alpha} P_{b} P_{c} e^{-\alpha L} \eta
$$

Where $\mathrm{n}$ is the refractive index of the fiber, $\lambda$ is the wavelength of information carrying signal, D is degeneracy factor and $P_{a}, P_{b}, P_{c}$ are power values of three input waves. $A_{e f f}$ is the effective area, $L_{\text {eff }}$ is the effective length, $\eta$ is the mixing efficiency.

The efficiency of FWM is given by [10]:

$\eta=\frac{\alpha^{2}}{\alpha^{2}+\Delta \beta^{2}}\left(1+\frac{\left.4 e^{-\alpha I_{\sin ^{2}}^{2}\left(\frac{\Delta \beta}{2}\right)}\right)}{\left(1-e^{-\alpha L}\right)^{2}}\right)$

Where $\Delta \beta$ is propagation coefficient and can be written as [10]:

$\Delta \beta=\beta_{a b c}+\beta_{c}-\beta_{b}-\beta_{a}$

Here, $\beta$ denotes propagation constant of light.

\section{Simulation Setup}

The main goal of this analysis is to determine how channel spacing in wave division multiplexing and chromatic dispersion coefficient affects the efficiency of FWM in an optical transmission system. The motive is to use the outcomes of the simulations to establish a feasible hardware implementation. Simulations are carried out using Optisystem 7.0 simulation software with a 4 channel WDM system using externally modulated laser sources on an optical fiber link of length $10 \mathrm{~km}$ at $1550 \mathrm{~nm}$ central wavelength. The simulation model used in the analysis shown in Figure 3 and Optisystem simulation setup is shown in Figure 4.

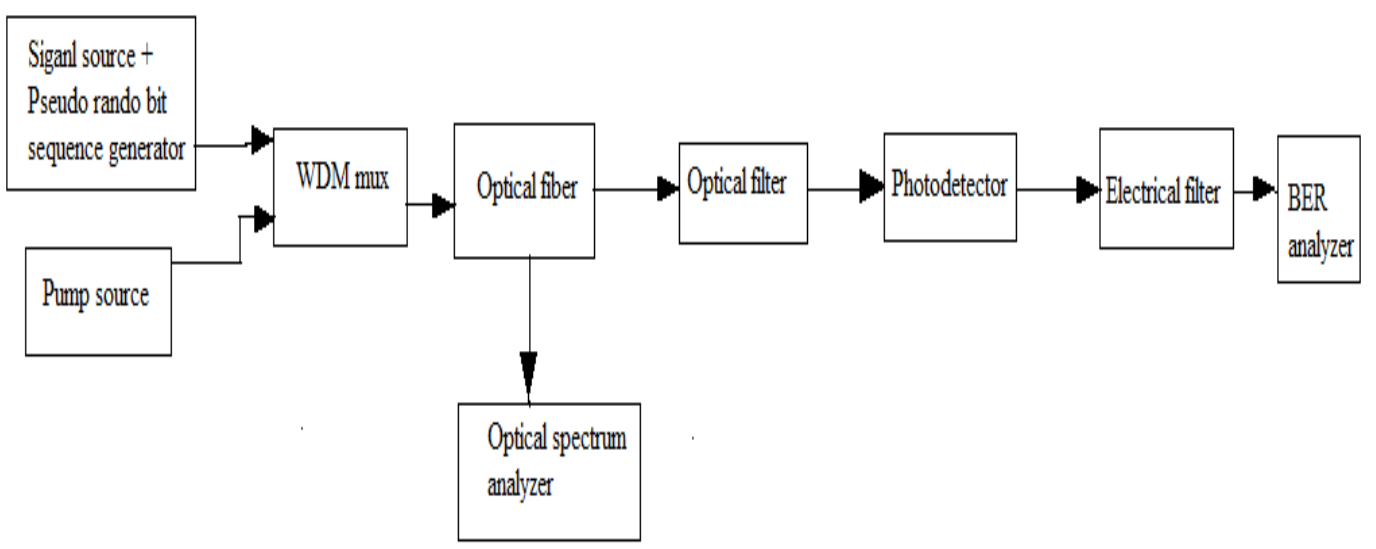

Figure 3. Simulation Model 


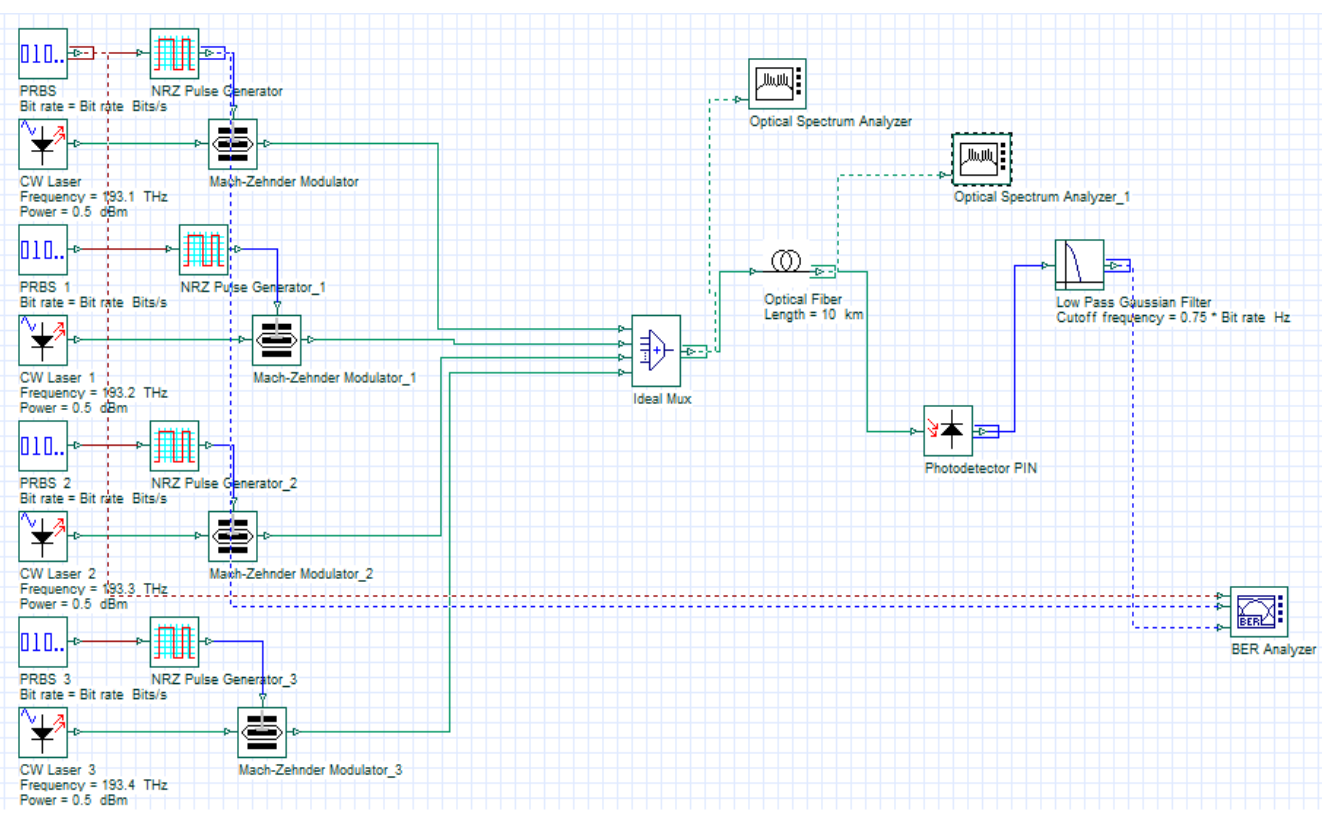

Figure 4. Simulation Setup

\subsection{Transmitter Section}

The transmitter section consists of a pseudo-random pulse generator which produces a stream of 0 's and 1's at a rate of 2.5 Gbps which are fed to the input of NRZ pulse modulator. NRZ pulse modulator converts this stream of 0's and 1's in the form of electric pulses having NRZ format. These electrical pulses from the output of NRZ pulse modulator are fed to the input of Mach-Zender modulator which modulates information signal with a continuous wave laser. The transmitter section consists of 4 such channels separated at certain frequency interval.

\subsection{Fiber Section}

In this simulation 4 WDM channels have been used which are separated from each other at certain frequency interval. These 4 channels are multiplexed and transmitted into a single mode fiber of length $10 \mathrm{~km}$. Various non-linearity such as four-wave mixing can be induced in the simulation using Optisystem 7.0 software. Different parameters such as chromatic dispersion coefficient, fiber length, and other dispersion parameters can be adjusted using Optisystem software.

\subsection{Receiver Section}

The optical signal from the output of the fiber is fed into the input of PIN photodiode which converts optical information signal into an electrical information signal. An APD photodiode can also be used in place of PIN photodiode for better performance. The output of PIN photodiode is fed to low pass Gaussian filter which removes all the highfrequency noise present in the received signal. At last signal is analyzed using BER analyzer. In order to analyze optical signal at different stages of the optical link, an optical spectral analyzer has been used to evaluate frequency spectrum of the signal.

\section{Results and Discussions}

In this paper, investigation has been done to analyze the effect of variation in WDM channel spacing and chromatic dispersion coefficient in the efficiency of FWM effect in optical wave division multiplexing systems using the above-presented simulation setup 
and simulation parameters. Optisystem 7.0 has been used to carry out different simulations and results have been evaluated on the basis of eye diagrams and input/output frequency spectrum of the signals. The various simulation parameters used in this simulation are given in Table no.1.

Table No.1

\begin{tabular}{|c|c|c|}
\hline Serial No. & Parameters & Value \\
\hline 1. & Reference Frequency (nm) & 1550 \\
\hline 2. & Fiber Length (km) & 10 \\
\hline 3. & Dispersion Coefficient (ps/nm/km) & $0-4$ \\
\hline 4. & Differential group delay (ps/km) & 0.5 \\
\hline 5. & Effective Area (um2) & 50 \\
\hline 6. & Attenuation $(\mathrm{dB})$ & 0.2 \\
\hline 7. & Laser Frequency (THz) & $193.1-193.4$ \\
\hline 8. & Filter Type & Gaussian \\
\hline 9. & Modulator Type & Mach-Zender \\
\hline 10. & Extinction Ration (dB) & 30 \\
\hline 11. & Symmetric Factor & -1 \\
\hline 12. & Bit Rate (Gbps) & 2.5 \\
\hline 13. & Transmitting Power $(\mathrm{dBm})$ & 8 \\
\hline 14. & No of Samples & 8192 \\
\hline 15. & Sample Bit Rate & 64 \\
\hline
\end{tabular}

The optical spectrum of the input signal when equal spacing has been used in all the channels is shown in Figure 5. It can be seen in the frequency spectrum of the input signal that there are four channels equally placed and there is no other frequency component present in the spectrum of the input of WDM transmission signal. 


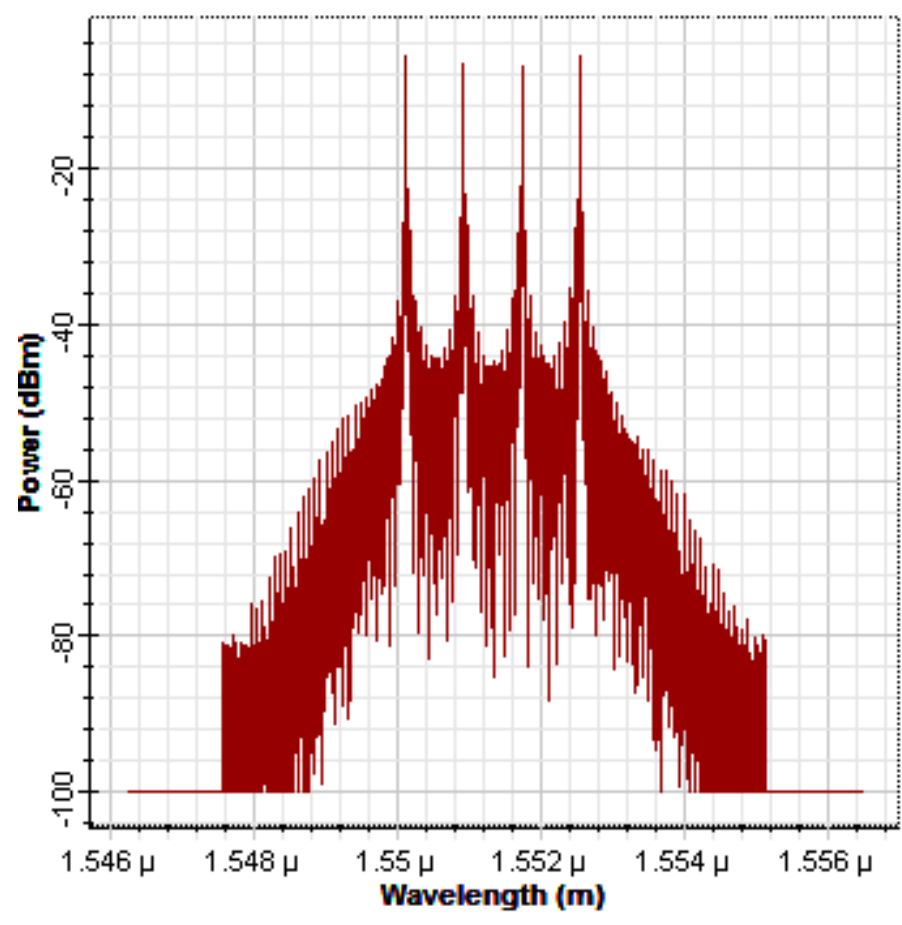

Figure 5. Input Signal Frequency Spectrum

The spectrum of output signals when dispersion coefficients are $0 \mathrm{ps} / \mathrm{nm} / \mathrm{km}, 0.4$ $\mathrm{ps} / \mathrm{nm} / \mathrm{km}, 4 \mathrm{ps} / \mathrm{nm} / \mathrm{km}$ are shown in Figure 5, Figure 6. Figure 7 and Figure 8 respectively.

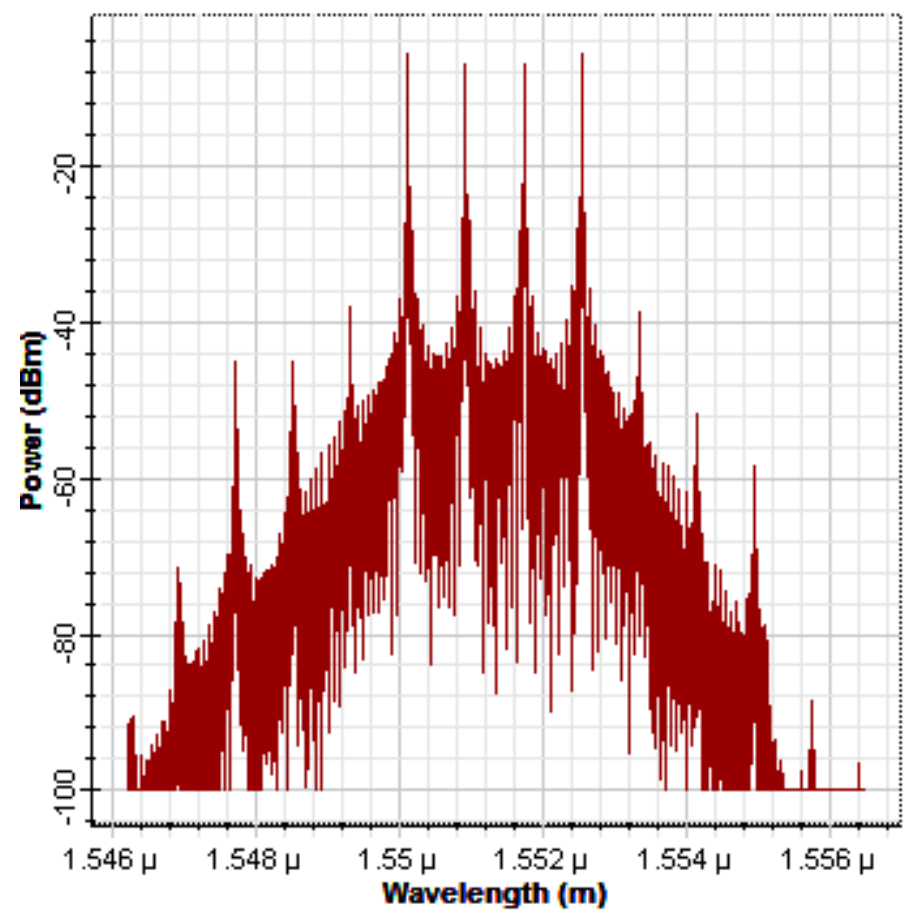

Figure 6. Output Signal Frequency Spectrum (D=0 ps/nm/km) 


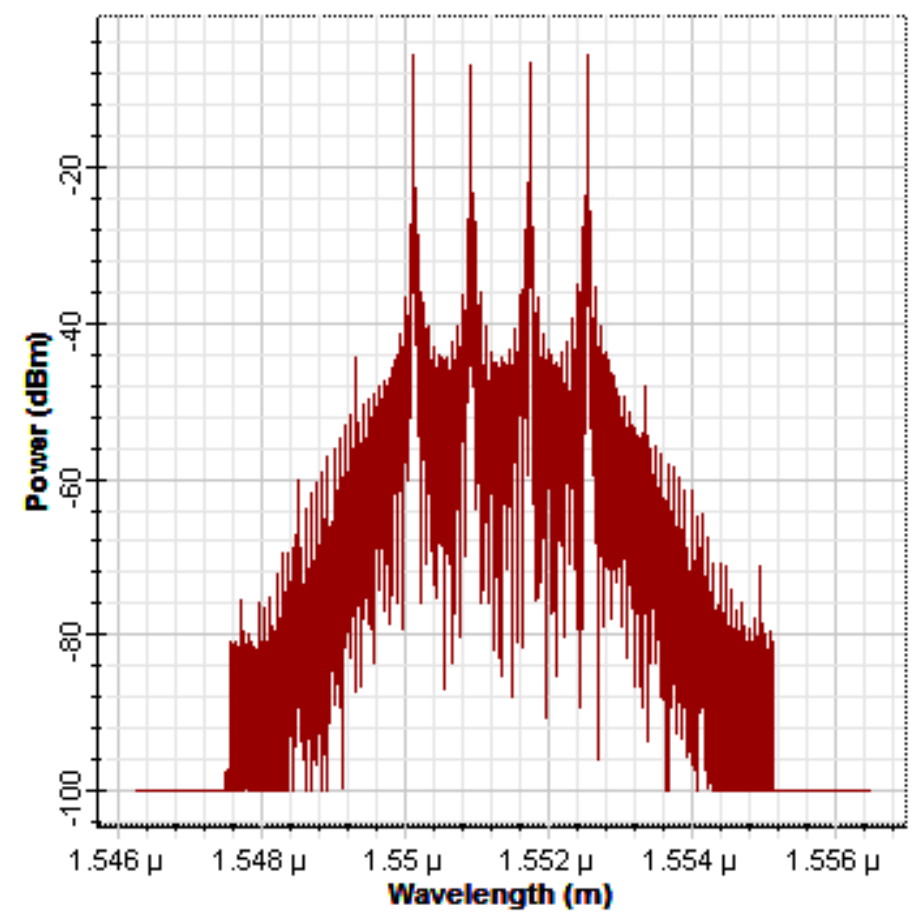

Figure 7. Output Signal Frequency Spectrum ( $D=0.4 \mathrm{ps} / \mathrm{nm} / \mathrm{km})$

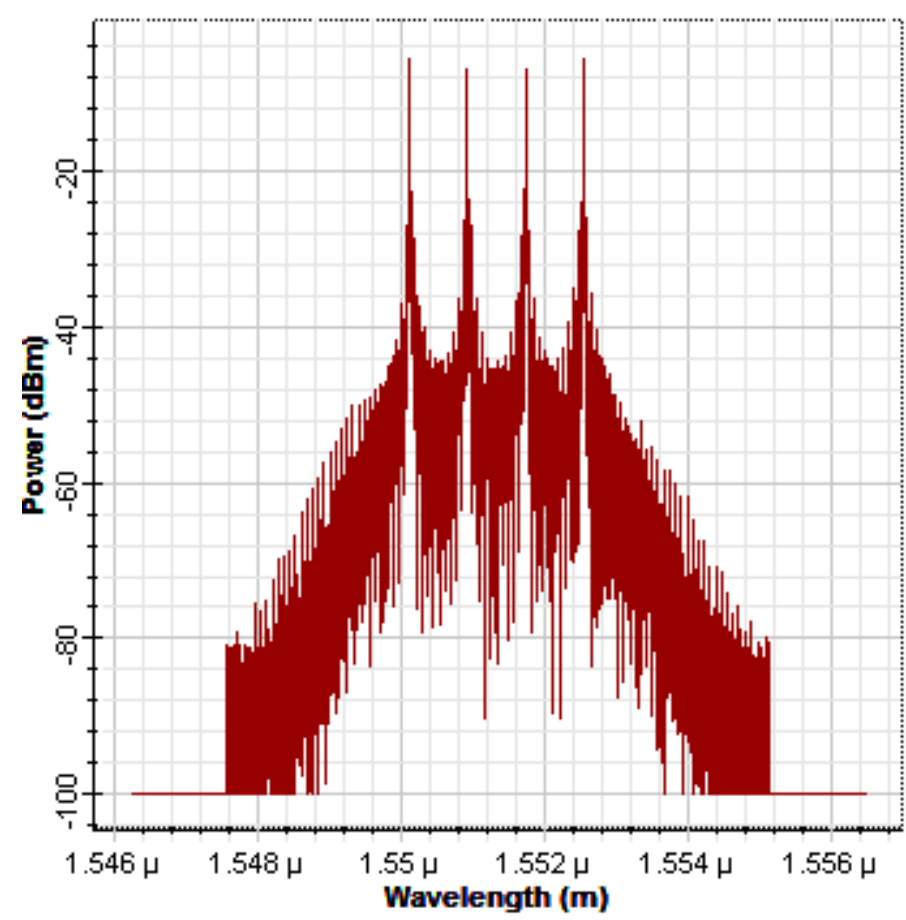

Figure 8. Output Signal Frequency Spectrum ( $D=4 \mathrm{ps} / \mathrm{nm} / \mathrm{km})$

From the above simulations results, it can be seen that due to four wave mixing effect some mix frequencies also known as beat frequencies are produced at the output of optical fiber. It can also be seen that as the value of chromatic dispersion coefficient is increased the power levels of beat frequencies is suppressed. When the chromatic dispersion coefficient is $0 \mathrm{ps} / \mathrm{nm} / \mathrm{km}$ significant side frequencies are generated which steal 
power from the original information carrying signal as shown in Figure 6 . When the value of chromatic dispersion coefficient is increased to $0.4 \mathrm{ps} / \mathrm{nm} / \mathrm{km}$ the power levels of side frequencies are reduced as shown in Figure 7. When the value of chromatic dispersion coefficient is further increased to $4 \mathrm{ps} / \mathrm{nm} / \mathrm{km}$ the side frequencies are suppressed to a large extent.

In this analysis, the effect of channel spacing on the efficiency of FWM has also been analyzed by observing eye diagrams for both equally spaced and unequally spaced channels as shown in Figure 9 and Figure 10 respectively.

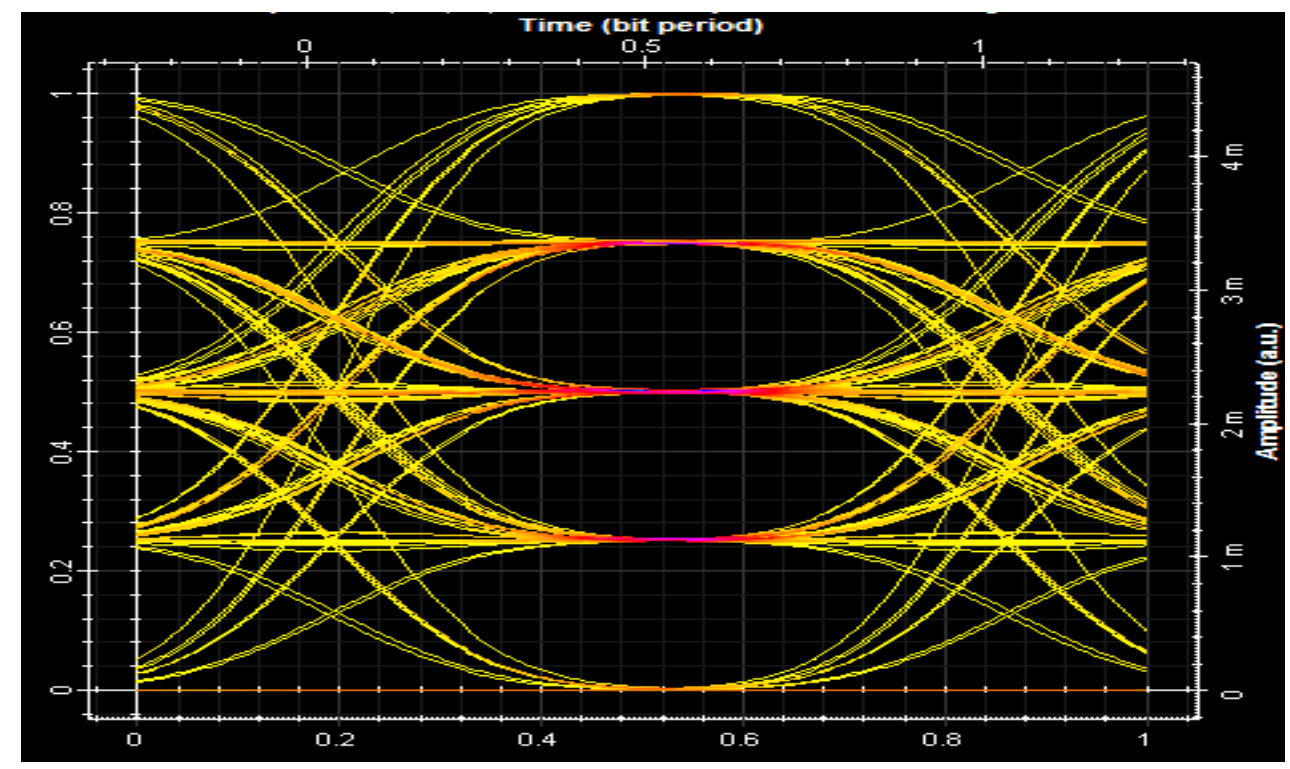

Figure 9. Eye Diagram for Equal Channel Spacing ( $D=0 \mathrm{ps} / \mathrm{nm} / \mathrm{km}$ )

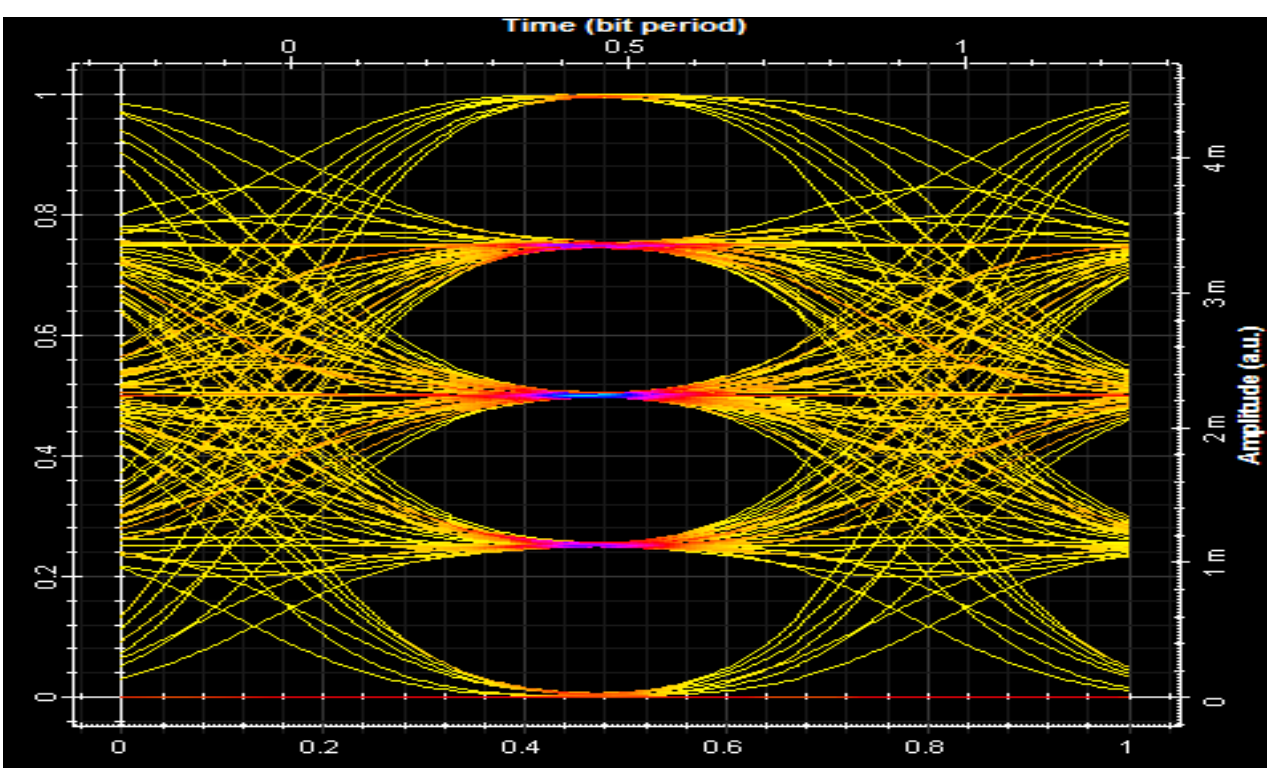

Figure 10. Eye Diagram for Unequal Channel Spacing ( $D=0 \mathrm{ps} / \mathrm{nm} / \mathrm{km}$ )

From the above simulation results, it can be seen that the efficiency of four-wave mixing is reduced slightly in case of unequal channel spacing and hence overall performance of WDM system is improved.

However further suppression of FWM frequencies can be achieved by increasing the value of chromatic dispersion coefficient as shown in Figure 11. 


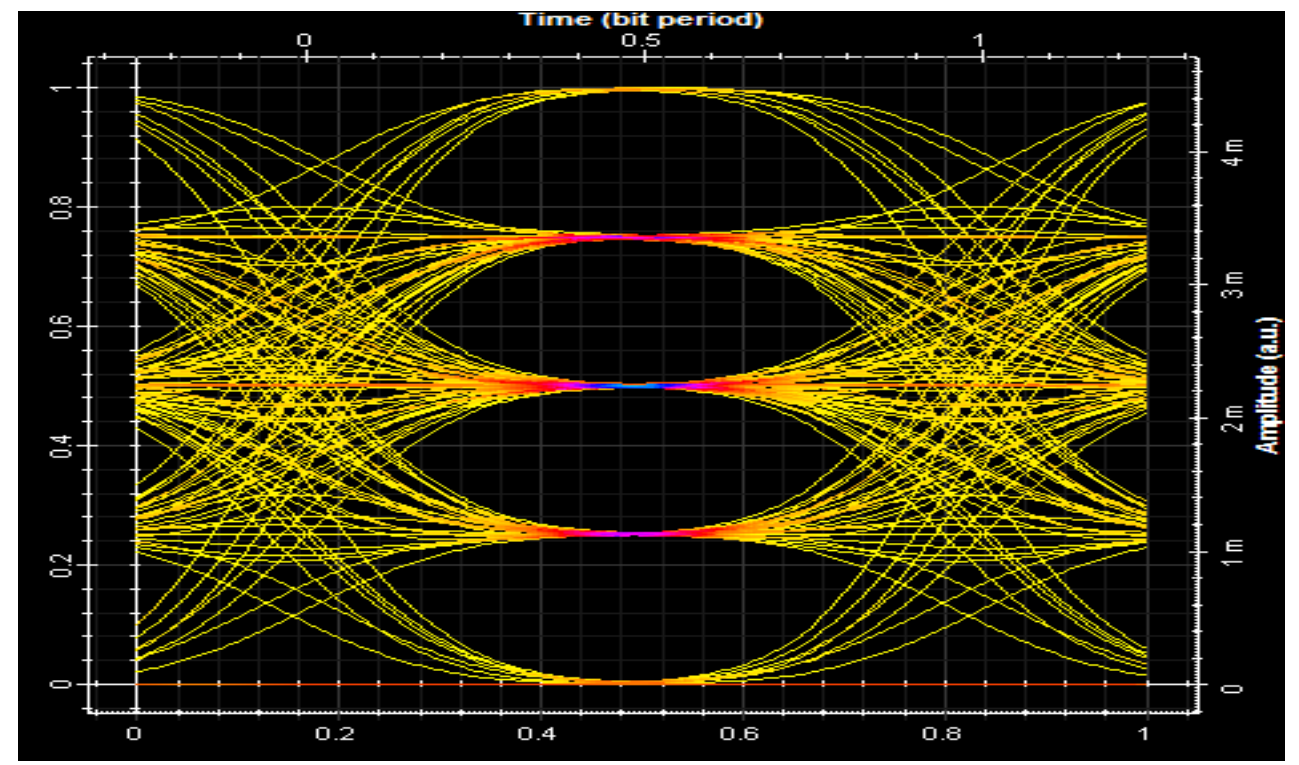

Figure 11. Eye Diagram for Equal Channel Spacing ( $=4 \mathrm{ps} / \mathrm{nm} / \mathrm{km})$

\section{Conclusion}

In this paper, the impact of channel spacing in WDM system and chromatic dispersion coefficient on the efficiency of FWM effect has been analyzed. From the results, it can be seen that as the value of chromatic dispersion coefficient is increased there is significant suppression of FWM frequencies and thus improvement in performance of WDM transmission system. Also, FWM effect in the case of unequal channel spacing is reduced to a great extent as compared to that of in equal channel spacing. So in order to improve the overall performance of a WDM transmission system, the unequal spacing in channels is recommended along with an optimum chromatic dispersion level.

\section{References}

[1] O. Aso, M. Tadakuma and S. Namiki, "Four-wave mixing in optical fibers and its applications", Furukawa Review, no. 19, (2000), pp. 63-68.

[2] P. L. Li, D. X. Huang, X. L. Zhang, J. Chen and L. R. Huang, "Theoretical Analysis of Tunable Wavelength Conversion Based on FWM in a Semiconductor Fiber Ring Laser", IEEE Journal of Quantum Electronics, vol. 41, no. 3, (2005) March, pp. 581-588.

[3] G. Charlet, E. Corbel, J. Lazaro, A. klekamp, W. Idler, R. Dischler and S. Bigo, "Comparision of System Performance at 50, 62.5 and $100 \mathrm{GHz}$ Channel Spacing over Transoceanic Distances at $40 \mathrm{Gcit} / \mathrm{s}$ Channel Rate using RZDPSK", Electronics Letters 3rd, vol. 41, no. 3, (2005) February.

[4] O. Aso, M. Tadakuma and S. Namiki, "Four-Wave Mixing in Optical Fibers and Its Applications", WP Team, Opto-technology Lab., R \& D Div.

[5] G. P. Agrawal, "Nonlinear Fiber Optics, Second Edition", Academic Press, San Diego, USA, Chap. 10, (1995).

[6] Yamamoto, "Optical Fiber Communications Technology", Nikkan Kogyo Shimbun, Chap.11, (1995).

[7] E. K. B. Kiang and S. P. Majumder, "Analytical Evaluation of the Impact of Four Wave Mixing on the Bit Error Rate Performance of a WDM Soliton Transmission Link", Journal of Optical Communications, vol. 23, no. 4, (2002), pp. 122-126.

[8] J. Zweck and C. R. Menyuk, "Reduction of intra-channel four-wave mixing using subchannel multiplexing", IEEE Photonics Technology Letters, vol. 15, no. 2, (2002), pp. 323-325.

[9] M. Kowalewski, M. Marciniak and A. Sedlin, "Nonlinear interactions in wavelength-multiplexed optical fibre telecommunication systems", Journal Opt. A: Pure Appl. Opt. 2, (2000), pp. 319-326.

[10] S. Shuxian, "Higher-order four-wave mixing and its effect in WDM systems", Optical Society of America, vol. 7, no. 4, (2000), pp. 166-171.

[11] H. G. Batshon, I. B. Djordjevic and B. V. Vasic, "An improved technique for suppression of intrachannel four-wave mixing in 40-Gb/s optical transmission systems", IEEE Photonics Technology Letters, vol. 19, no. 2, (2007), pp. 67-72. 


\section{Author}

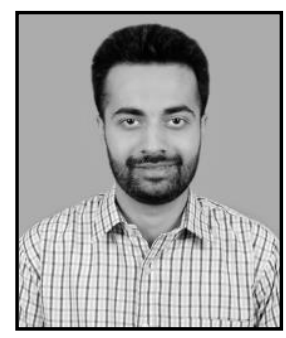

Mehtab Singh did his B.E in Electronics and Communication Engineering from Thapar University, Patiala, India in the year 2013 and M.Tech in Electronics and Communication Engineering with specialization in Communication Systems from Guru Nanak Dev University, Amritsar, India in the year 2015. Presently, he is working as an assistant professor in the department of Electronics and Communication Engineering, Satyam Institute of Engineering and Technology, Amritsar, India. His areas of interests include Optical Communication and Wireless Communication. 
International Journal of Signal Processing, Image Processing and Pattern Recognition Vol.8, No.11 (2015) 